\title{
EXPERIMENTAL RESEARCH ON THE COURSE OF THE OPTIC FIBRES.
}

\author{
BY GEORGE DEAN, M.B., AND C. H. USHER, M.B. \\ (Second Communication.)
}

IN a previous communication to the Ophthalmological Society ${ }^{1}$ we reported results obtained by us in rabbits, which showed that a degeneration of the optic nerve resulted from a lesion of the retina; that the degeneration had a limited distribution, occupying in the main the same position in relation to the nerve section throughout the whole length of the nerve, and that the position of the degeneration in the sectional area of the nerve corresponded with the part of the retina wounded. The degeneration in the optic nerve resulting from a macular lesion in the monkey was also described.

The lesions of the retina were made by a Graefe knife. Marchi's osmium-bichromate method was used for demonstrating the degeneration. For the details of the methods of operation, fixation, hardening, and staining, we must refer to the previous communication.

In the present communication we propose to give the results of other experiments on monkeys in which degeneration $(a)$ in the optic nerve, and $(b)$ in the chiasma, resulted from retinal lesions or division of an optic nerve. The method of operation on the retina was the same as that adopted in the case of the rabbits, namely, entrance of a Graefe knife at the posterior part of the ciliary region, guidance of the point of the knife, aided by the ophthalmoscope, and incision of the selected part of the retina, or the

' Vol. xvi. of the Transactions. Please note that figs. 52 and 62 are drawn as seen by the indirect method, and " $D$ " and " $V$ " should be transposed in fig. 67 . 
production of a lesion by means of a fine thermo-cautery, as in the experiment on the monkey described in our last communication.

The methods used for hardening and staining the tissues, and also those for keeping the optic nerves from rotating, were the same as those described in the previous paper.

A complete series of transverse vertical (coronal) sections was made of the chiasma in each case.

The cases dealt with in the present communication can be conveniently divided into three groups:-

(I.) Division of an optic nerve.

(II.) Retinal lesions at a distance from the macula.

(III.) Retinal lesions at the macular region.

\section{Group I.-Division of an Optic Nerve.}

In each case the nerve was divided in the orbit. Attempts were made to accomplish this in such a way as to do a minimum of damage to the blood-vessels.

\section{Monkey 1 (Macacus Rhesus).}

In this case the left optic nerve was divided transversely far back in the orbit. The animal lived only eight days, and the cause of death was uncertain.

No ophthalmoscopic examination was made after the operation.

Microscopical examination of the optic nerves.-In front of the incision there is well-marked degeneration as evidenced by numerous black points throughout the section. Behind the incision there is little or no evidence of degeneration; no black points can be made out.

\section{Monkey 2 (Macacus Rhesus).}

The right optic nerve was divided far back in the orbit. The animal was killed after thirty-two days.

Ophthalmoscopic examination before killing the animal : optic disc pale, retinal vessels not easily made out.

Microscopical examination.-In front of the incision there is a considerable amount of new fibrous tissue and comparatively little staining by osmic acid. There is an 
entire disappearance of the normal relation of the framework of the nerve and its nerve bundles. Behind the incision there is dense staining by osmic acid throughout the sections.

\section{Monkey 3 (Macacus Rhesus).}

The right optic nerve was divided far back in the orbit. Immediately after operation the retinal arteries were reduced to the size of fine threads. Retina somewhat hazy. Retinal veins not markedly changed. The animal lived tifteen days.

Microscopical examination.-In front of the incision the vessels of the septa are very prominent, and the septa distinct. There are numerous black points throughout the whole area of the nerve. It was observed that the transverse diameter of the nerve was greater in front than behind the incision.

Behind the incision there is a diffuse well-marked degeneration containing more densely-packed black points than in front of the incision.

Chiasma.-On tracing the degeneration from before backwards it is found in front to be almost confined to the right half; on the ventral aspect, however, there is a narrow tract passing to the left side.

Further back the crossing degeneration is much more extensive, and is arranged in distinct transverse columns. Still further back other degenerated columns appear and occupy a region nearer the dorsal surface, and there is a widespread degeneration on both sides of the chiasma, but the degeneration remains much less marked in the left lateral region.

On the right side of the chiasma posteriorly, and occupying a ventral position, is an area presenting very few black points; the corresponding area on the left side is densely packed with them.

\section{Monkey 4 (Macacus Rhesus).}

The left optic nerve was divided far back in the orbit. Immediately after operation the optic disc was pale, and the retinal arteries much narrower than those of the other eye.

The animal lived thirty-one days. 

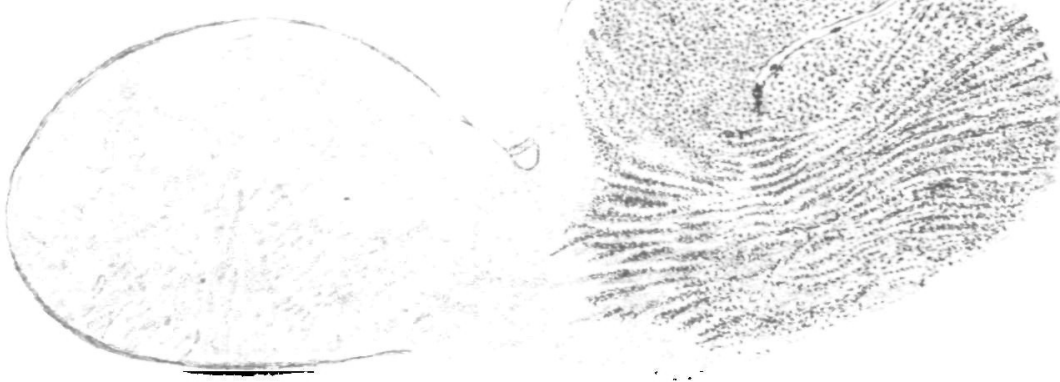

3

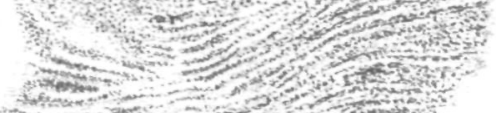

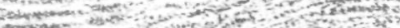

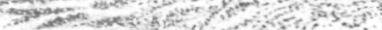

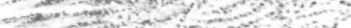

soper and

-

Fig. 1 .

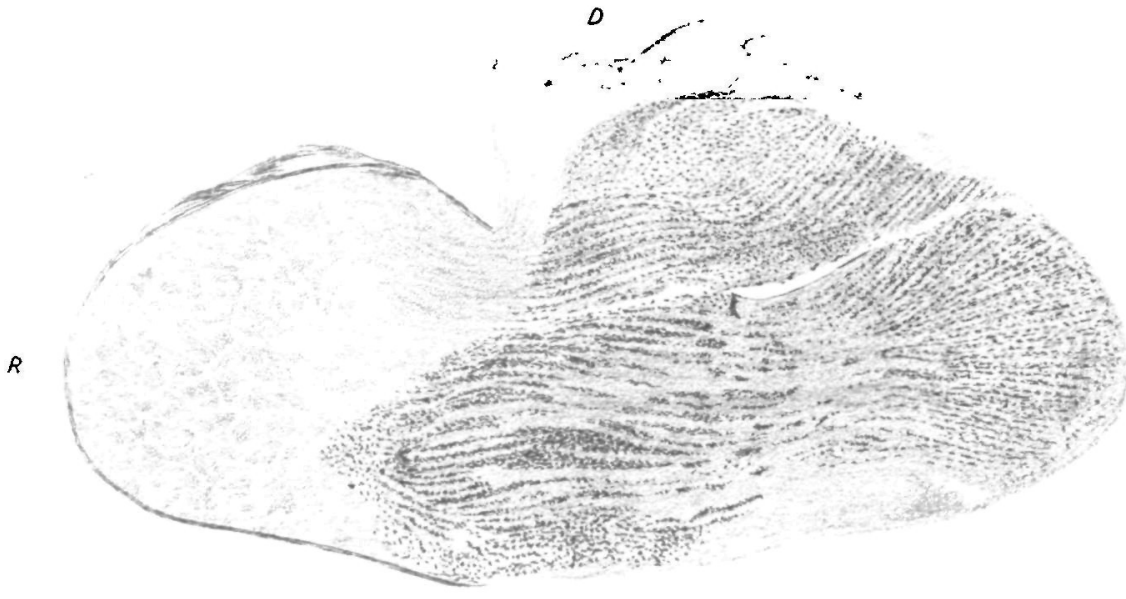

V

Fig. 2 . 
Microscopical examination.-Behind the scar there is a dense diffuse degeneration. In front of the scar there is a degeneration, but the black points are smaller than behind the scar. The normal relation of the fibrous tissue to the bundles is destroyed, the angular character in these having disappeared, the bundles being now rounded in outline.

Chiasma (Plates I. and II.).-In the front part of the chiasma the degeneration begins to cross at once on the ventral aspect. Further back it encroaches more and more on the right side, most of it still lying in the ventral part. The black points are here arranged in transverse bands. The crossing degenerated fibres become more numerous and fill more and more of the dorsal part.

Towards the posterior part of the chiasma a ventral median area on the same side as the operated nerve becomes free from degeneration, and further back this area increases in extent. The corresponding area on the other side is densely packed with black points. In sections from further forward in the chiasma this region (ventral median) contains fewer degenerated fibres. The outer part of the chiasma on the side of the unoperated nerve is practically free from black points. The outer part on the other side is full of black points.

\section{Monkey 5 (Macacus Rhesus).}

This was an enucleation of the right eyeball. The animal lived thirty days.

Microscopical examination.-There is well-marked degeneration throughout the nerve.

Chiasma.-The crossing of the degeneration in the chiasma commences on the ventral part anteriorly, and on passing back gradually involves an increasing area of the left side. At the posterior part of the chiasma on the right side the ventral median area contains very few black points. The corresponding area on the opposite half of the chiasma is very full of black points. The degeneration is much less marked at the outer part of the left balf. Posteriorly no marked difference as to the number of degenerated fibres can be made out between the two sides. 
Grodp II.-Retinal Lesions away from the Macola.

$$
\text { Monkey } 6 \text { (Macacus Rhesus). }
$$

An incision was made in the right eye below the macular region with a Graefe knife.

Ophthalmoscopic appearance immediately after withdrawal of the knife.-There was a bright red area, one and a quarter discs' diameter in length, and its breadth one-sixth of its length. It had well-defined margins, its long axis was vertical, and was so situated that its upper end would have touched a horizontal line drawn through the lower margin of the optic disc. It lies one optic disc's diameter external to the optic disc.

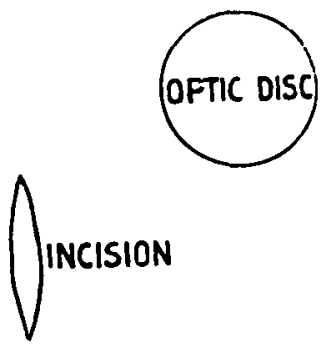

Right Eye.

The animal lived for twenty-nine days.

Microscopical examination of the right optic nerve.Throughout the entire length of the nerve there is a wellmarked degeneration situated ventrally. It is roughly triangular, its base being at the periphery.

In the anterior part of the chiasma a degeneration is present in the ventral part of the right side. The bulk of the degeneration retains this position and situation, but at the posterior part of the chiasma a few of the fibres have crossed.

\section{Monkey 7 (Macacus Rhesus).}

In the left eye a similar incision was made with a cataract needle in the same way as was done with the Graefe knife in the previous case. 
Ophthalmoscopic appearance on withdrawal of needle.A bright red area with well-defined margins was seen; its length was about half an optic disc's diameter. It lay one and a quarter discs' diameter below the yellow spot.

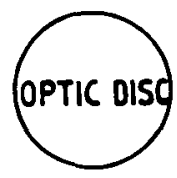

\section{MACULA \\ LUTEA}

\section{INCISION}

Left Eye.

The animal was killed after thirty-one days.

Microscopical examination of the left optic nerve.-There is found throughout the whole length of the nerve a degeneration with the following characters :-

It occupies a well-defined area in the ventral part of the right ventral quadrant. This area contains a small number of degenerated fibres; it lies chiefly at the periphery, and comes in contact with about one-twelfth of the circumference of the nerve. Some of the black points extend to half way between the central vessels and the edge of the nerve.

Chiasma.-In the front part of the chiasma the degeneration lies ventrally in the left half, and is close to the periphery. There is no degeneration in the right half at this level. Further back, the degeneration passes further outwards and is more diffuse. A few black points, however, are seen nearer the centre of the chiasma, and there are now a few in the right half of the chiasma which become more numerous in the sections further back.

$$
\text { Monkey } 8 \text { (Macacus Rhesus). }
$$

A lesion of the right retina was produced by a Graefe knife.

Ophthalmoscopic examination immediately before the death of the animal.-The lesion was seen at the inner part 
of the fundus at a distance of one and a balf optic discs' diameter from the inner edge of the optic disc. Its long axis was vertical and measured balf an optic disc's diameter, its breadth was the sixth of its length. The lesicn was very white, and its outer margin was bordered with black pigment.
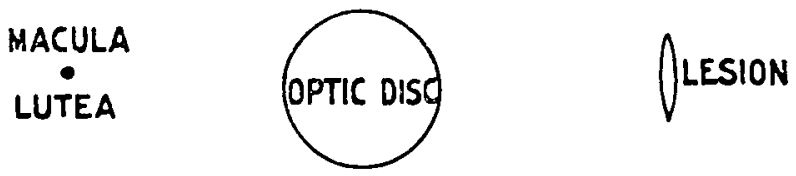

Right Eye.

Microscopical examination of right nerve.-There is a beautifully marked degeneration at the inner part of the nerve, throughout its whole length. Anteriorly it is wedgeshaped with its base at the periphery, posteriorly it is less triangular and forms a narrow band, lying close to the inner margin of the nerve.

Chiasma.-In its anterior part the degeneration lies wholly in the right balf, close to the uniddle line, which is here distinguished by a septum. (Plate III.)

Further back the black points gradually cross to the other side in distinctly defined bands. (Plate IV.)

At the posterior part of the chiasma the degeneration has entirely crossed to the left side.

\section{Monkey 9 (Bonnet Monkey).}

A vertical incision was made at the outer part of the left eye near the equator. In this case the method of operation differed from that employed in previous cases. The lesion of the retiua was produced by puncturing the coats of the eye with a Graefe knife at the site selected so that the vitreous had not to be traversed in making the lesion.

After death the globe was opened and a distinct linear lesion about $2 \mathrm{~mm}$. long was found at its outer part in the equatorial region. The vitreous showed nothing abnormal.

The animal was killed thirty-five days after operation. 

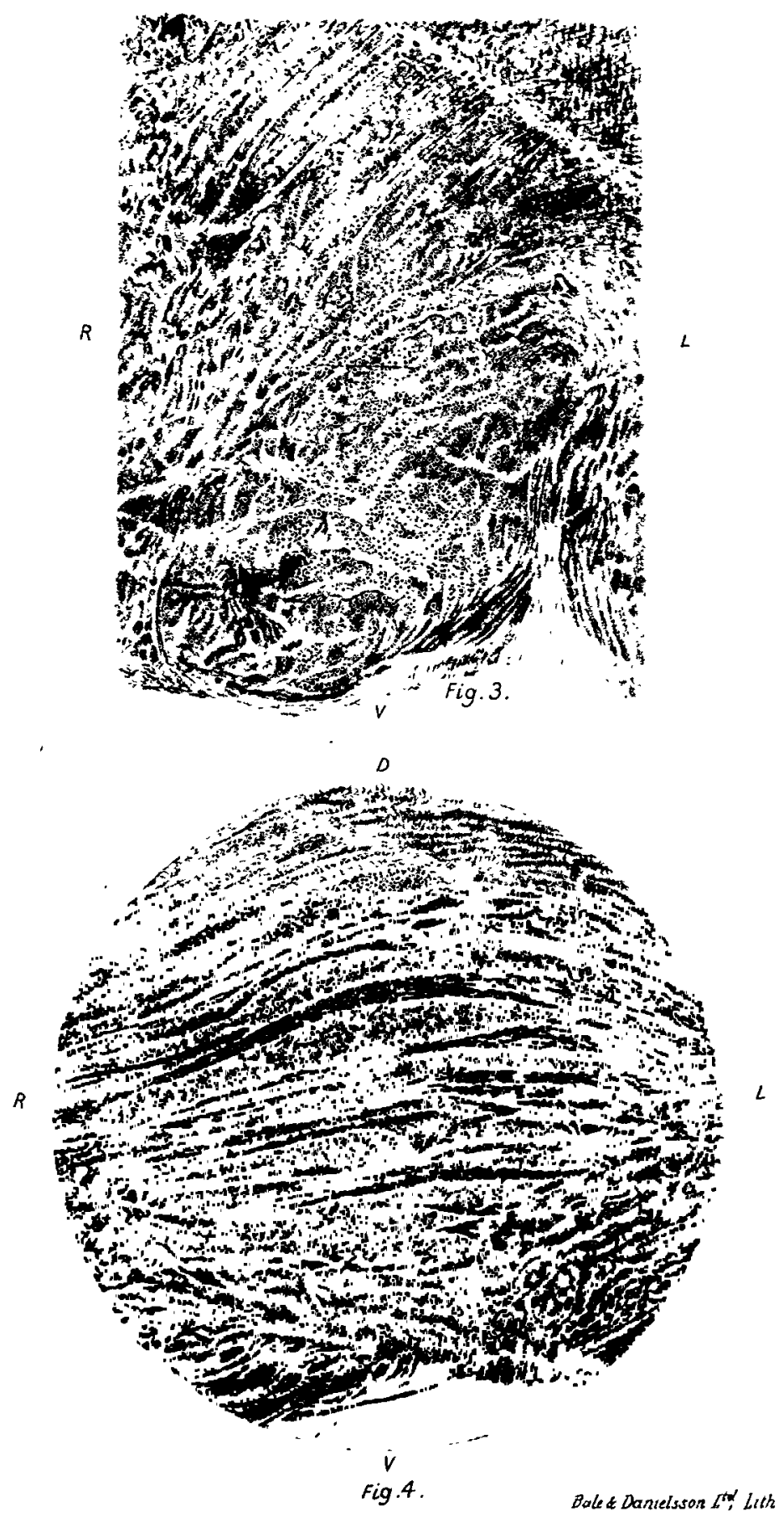
Microscopical examination of the left nerve.-A degeneration is found in the outer dorsal part of the nerve throughout its whole length. The rest of the nerve is free from points of degeneration.

Chiasma.-The sections from the anterior part of the chiasma cut badly and could not be made use of. Those about the middle of the chiasma show that most of the degeneration lies in the left half near the lateral periphery, but there are also some black points in the ventral part of the right balf.

Passing backwards the degeneration becomes less in the left side of the chiasma and approaches the middle line, whilst it becomes more marked at the ventral part of the right half of the chiasma. Definite bands of degenerated fibres are seen running close to the ventral periphery from the one side to the other.

At the most posterior part only a very few points of degeneration are left in the operated side, the bulk of them baving crossed.

\section{Group III.-Retinal Lestons at the Macular REgION.}

Monkey 10 (Macacus Rhesus).

The facts in regard to this experiment in so far as the nerve was concerned were published in our previous communication, but we may bere briefly recapitulate them.

A lesion was made in the retina of the right eye between the optic disc and the yellow spot by means of a fine thermo-cautery. There was found at the anterior part of the nerve a well-defined area of degeneration, triangular in outline, with its base at the outer periphery, its apex close to the central vessels. The area occupied about one twelfth of the nerve-section. In the middle and posterior parts of the nerve the degeneration lay in the centre of the nerve, no part reaching the periphery.

The chiasma, which has since been examined, shows the degeneration on the same side as the lesion, quickly 
reaching the dorsal periphery towards the middle line. The degeneration extends towards the opposite side along the dorsal periphery. (Plate V.) Near the centre of the chiasma about half the degenerated fibres have crossed, and at the posterior part nearly all have reached the other side, retaining, however, their dorsal situation.

\section{Monkey 11 (Macacus Rhesus).}

The lesion in this case was produced in the left eye by the thermo-cautery.

Examined ophthalmoscopically immediately after the operation the fundus showed a large hæmorrhage with welldefined margins extending from the optic disc to about one optic disc's diameter external to the yellow spot. Its breadth is equal to two or three discs' diameters. The right fundus was found to be normal.

The animal was killed by chloroform thirty-one days after the operation.

Microscopical appearance of left optic nerve.-In the anterior portion of the nerve at its outer side there is a well-marked degeneration, which is wedge-shaped, with the apex at the central vessels and with the base at the periphery.

Towards the middle of the nerve the degeneration comes to lie near the centre, but is more in the ventral than in the dorsal half of the sections. It maintains this position throughout the rest of the nerve.

Chiasma.-In the front part the degeneration occupies the same relative position as it did in the posterior part of the left nerve. Further back it passes towards the centre of the chiasma and becomes more dorsal. Although the coarser black points do not reach the dorsal margin numerous finer points are scattered in this region.

More posteriorly so many of the degenerated fibres have passed over that about equal numbers lie on both sides of the central part of the chiasma.

Just in front of the optic tracts the degeneration is found to have completely crossed to the right side. 


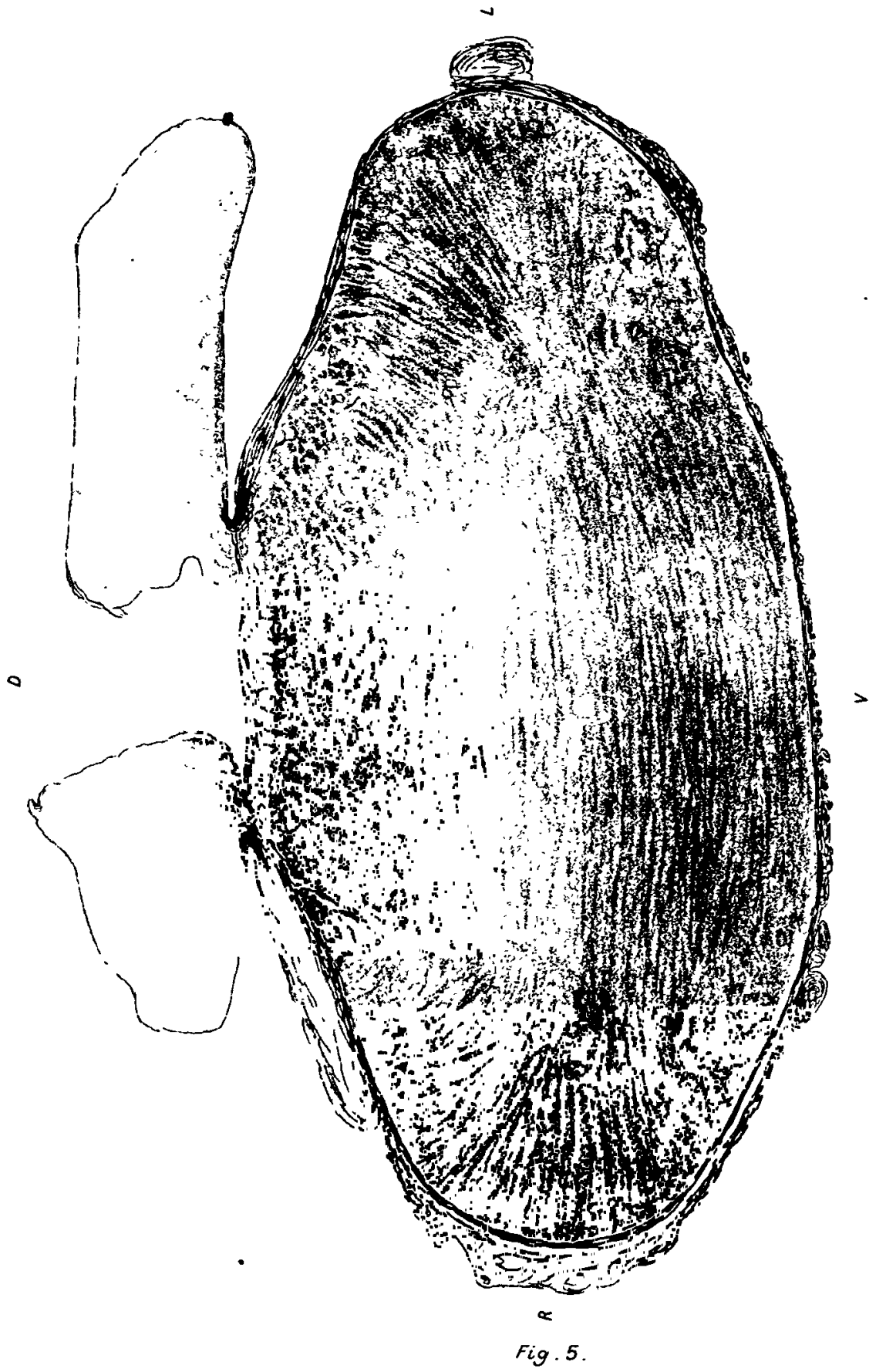

Bale \& Danuelsson $L^{\prime d}$, Lilh 


\section{Monkey 12.}

In this experiment a well-defined lesion was produced between the yellow spot and the optic disc. The degeneration was found to occupy the same position in the nerve as in the two previous experiments. No other black points were found elsewhere in this nerve.

Owing to tilting of the chiasma during embedding, the examination was not quite so satisfactory as in the two previous macular cases, but all the facts observed tend to confirm the results obtained in these.

In connection with this group we would record the following case :-

\section{Monkey 13.}

The condition of the fundus described in this monkey was not the result of operation, but was observed during an ophthalmoscopic examination previous to an intended experiment. The animal was a male Macacus Rbesus.

The outer part of both optic discs was found to be markedly pale. The lamina oribrosa was conspicuous in this situation. The whole appearance presented a marked contrast to that of the discs of the monkeys which we had previously examined. The rest of the fundi, including both yellow spots, appeared normal, and the media were clear.

Microscopical examination of the optic nerves.-A tract of degeneration extends along the whole length of both optic nerves and through the chiasma to the optic tracts. The area occupied by this tract at the front of the nerves is triangular, with its base at the outer periphery, its apex at the central vessels. Further back in the nerves it becomes circular, and attains a central position, which it maintains as far as the chiasma. Anteriorly in the chiasma the degenerated fibres still occupy the central area of both halves. Soon after entering the chirsma they show a tendency to pass towards the dorsum, even before any crossing bas taken place. (Plate VI.) The first crossing of these fibres occurs at the dorsal periphery (Plate VII.), and as the sections proceed backwards the crossing degenerated 
fibres extend from the dorsal surface more and more ventrally until the affected area occupies the dorsal twothirds of the central part of the chiasma. (Plate VIII.) The degeneration never reaches the ventral periphery.

The degeneration occupies about one-third of the sections in the nerve, and at least that proportion in the chiasma. The affected tract throughout its whole extent is very well defined, and can be easily followed. The structure of the degenerated tract consists largely of newly-formed fibrous tissue. In sections stained by Marchi's method the affected area contains a fair number of well-defined black points. No gross lesion could be found in the nervous system to account for this condition.

Microscopical examination of the nerves on the unoperated side.-A microscopical examination was made of all the nerves from the side of the unoperated eye. In every case small black points were observed. This was in accordance with our previous experience, which had been obtained for the most part from experiments on rabbits. Various hypotheses to account for these points were discussed in our previous paper.

With the view of attempting to elucidate the difficult question of the origin of these points we carried out a number of tests on the following lines :-

Sections from the front and back parts of the nerves were examined and compared with the view of finding whether the points were more numerous towards the eye-ball or towards the chiasma. The idea was that if they were due to myelin drops in process of transportation from the nerve, with the lesion to the nerve on the unoperated side, they would probably be more numerous posteriorly. No difference, however, could be ascertained between the two parts.

Comparisons were instituted between these nerves obtained from cases where there were marked lesinns, such as division of the nerve, and those in which the lesions were very small. The object of this examination was to ascertain whether there was a correspondence between the number of black points in the nerve of the unoperated eye and the extent of the lesion on the operated side. If out of a series 


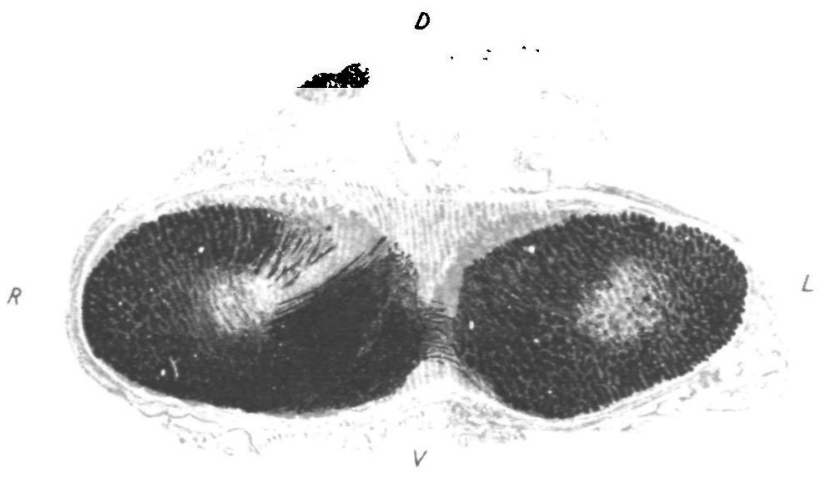

fig. 6.

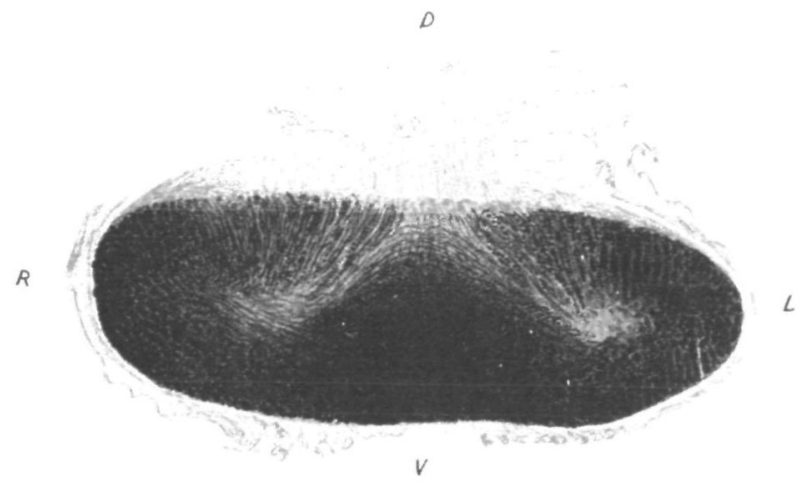

Fig. 7.

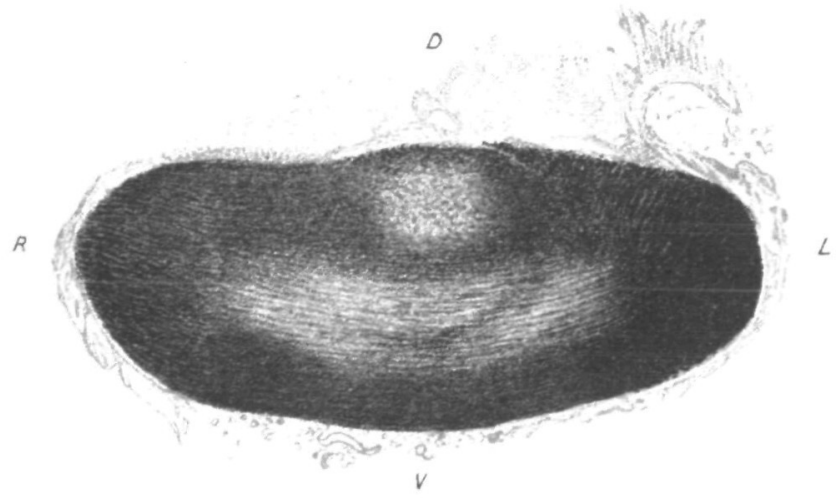

Fig. 8 .

Bale \& Durselsson Ltd Lith 
of specimens which were unknown to the observer at the time, a selection was made of those containing most black points, the slides selected were generally, but not invariably, found to belong to the cases where the optic nerve had been divided.

The points are always distributed throughout the sec.tions; we have never been able to determine the existence of definite groupings. The whole question is an extremely difficult and uncertain one, and though we have had more material at our disposal we are able to add nothing to our remarks in the Ophthalmological Transactions. ${ }^{1}$ None of the optic nerves on the uninjured side in our cases show so many, or such large black points as are represented by Parsons in his paper. (Plate II.) In this connection it may be mentioned that Parsons employed Busch's FormolMarchi method.

For a résume of the difficulties attending the use of Marchi's method we may refer to Spielmeyer's recent paper. ${ }^{2}$

Remarks and Conclusions.

Group I.

(Division of an optic nerve, five cases.)

Results obtained in the Optic Nerve.

The experiments in this group were made partly with the object of ascertaining whether any difference existed in the characters and extent of the degeneration in the parts of the nerve above and below the scar.

In three of the cases degeneration, as indicated by osmic acid staining, was most marked above the scar. In these cases, however, there were below the scar profound changes which involved largely the connective tissue framework and its relation to the nerve bundles. In this part of the nerves osmic acid staining was a much less marked feature than

1 Ref. loc. cit.

2 Walther Spielmeyer, "Die Feblerquellen der Marchi"schen Methode," Centralbl. $f$. Nertenheilkunde, xxv., July 15, 1903. 
in the part of the nerves above the scar. It appeared to us that a considerable amount of the difference in appearance above and below the scar was due to interference with the vascular supply, as was suggested by the ophthalmoscopic appearances, more especially in cases of Monkey 3 and Monkey 4.

In Case 1, which lived for only eight days after operation, the degeneration as evidenced by black points was extremely well marked below the incision, but scarcely at all above the incision.

\section{Results obtained in the Chiasma.}

(1) The degenerated fibres begin to cross over as soon as they enter the chiasma.

(2) The first crossing is seen to take place at the ventral periphery, due probably to the first junction of the optic nerves occurring ventrally. As the degenerated fibres proceed backwards they invade more and more of the chiasma from the ventral to the dorsal surface, till ultimately they are distributed over the whole central part of the chiasma.

(3) Only a few, if any, of the crossed fibres appear to approach the lateral periphery of the half of the chiasma corresponding to the unoperated nerve, whereas on the corresponding area of the opposite side the degeneration is dense.

(4) It could not be determined that the crossed fibres at the posterior part of the chiasma were more numerous than the uncrossed.

(5) A very definite tract of crossed fibres lies at the ventral part of the chiasma posteriorly.

Wieting ${ }^{1}$ examined the optic nerves, chiasma, and tracts of three cases in which there was complete atrophy of one of the optic nerves. He stained the sections by WeigertPal's method and found partial crossing of the fibres in the chiasma. The crossed fibres occupied a ventral median area which increased in size towards the posterior part of the chiasma.

' Wieting, " Zur Anatomie des menschlichen Chissma," Arch. f. Ophth., 1898, Bd. zlv., Abt. i., S. 75. 
Dimmer ${ }^{1}$ investigated two cases by Marchi's method. First case:-Enucleation of an eye, whose optic nerve was normal, six weeks before death. Second case :-Complete amaurosis of one eye and temporal hemianopia of the other, brought about by a tubercular growth of the chiasma. $\mathrm{He}$ found the crossed bundle lying chiefly in the ventral median part of the chiasma. It may be noted that the sections of the chiasma of his first case, figured in his paper, present a striking resemblance to the chiasma sections in our Group I.

\section{GroUP II.}

(Retinal lesions at a distance from the macula, four cases.)

Results obtained in the Optic Nerve.

The results in all the experiments as regards the degeneration are in accordance with those obtained by us in rabbits. The degeneration lies in the part of the sectional area of the nerve corresponding to the site of lesion in the retina. Experiments 6 and 7 are almost identical. In both the retinal lesions were below the macular region. The degeneration occupies a ventral position throughout the nerve and chiasma, and in both cases there is a slight crossing of the degenerated fibres.

\section{Results obtained in the Chiasma.}

(1) A ventral degeneration in the nerve (Cases 6 and 7) maintains a ventral position in the chiasma. A very few degenerated fibres cross to the other side.

(2) A lesion at the inner part of the fundus (Case 8) is followed by a degeneration at the inner part of the nerve which, in the front part of the chiasma, lies on the same side, but ultimately crosses completely to the opposite side.

(3) A very peripheral lesion in the outer part of the fundus (Case 9) is followed by a degeneration in the outer dorsal part of the nerve. In the chiasma the degeneration lies on the same side close to the lateral periphery,

'Dimmer, “Zur Lehre von den Sehnervenbahnen," Arch. f. Ophthal. 1899, Bd. xlviii., Abt. iii., S. 473.

VOL. XXVI. 
ultimately crossing and occupying the ventral periphery of the opposite side, only a few fibres remaining uncrossed at the posterior part of the chiasma. This experiment makes it probable that in this particular monkey (a specimen of the Bonnet Monkey), the great majority of the fibres cross in the chiasma. It is possible that this indicates a reversion to the lower or more primitive type of chiasma. Such a view is suggested by Schäfer ${ }^{1}$ to account for certain reported human cases which can be explained only on the bypothesis of complete crossing of the fibres. It may be noted, however, that Parsons (BraIN, Autumn Number, 1902, part xcix.) in two cases of temporal lesion, one in a Bonnet Monkey, the other in a Macacus Rhesus, found that a certain number of degenerated fibres were present in the opposite tract.

\section{GROUP III.}

(Retinal lesions at the macular region, three cases.)

Before giving a summary of our conclusions as to the course of the macular fibres in the optic nerve and chiasma, based on Case 13 and on the experimental cases in this group, it may be desirable to give a brief statement of the most important work on the subject of the course of the macular fibres.

Henschen ${ }^{2}$ studied clinically and pathologically a large amount of human material and compared his results with the cases recorded in the bibliography, and gives his conclusions regarding the localisation of the bundles in the optic nerve, chiasma, and tract. He states that the bundle from the macula lutea on each side runs in the central part of the optic nerve, and maintains its central position in the optic chiasma and in the optic tract.

Vossius, ${ }^{3}$ in a case of symmetrical central scotoma in

'Scbäfer, "Quain's Anatomy," vol. iii., pt. i., p. 118.

2 Henschen, "Klinische und anatomische Beiträge zur Pathologie des Gehirns," Zweiter Theil, Upsala (1892), S. 217, ref. Barker, "The Nervous System," p. 793.

- Vossius, Arch. für Ophthalm., xxviii., 3, 201. 
man, found that the macular fibres lie close under the dorsal margin of the chiasma. It will be noted that Case 13 reported by us corresponds very closely with Vossius' description and drawings.

Bunge ${ }^{1}$ found that the papillo-macular bundle maintained a dorsal position throughout the chiasma in man.

As having a practical bearing on the question of the exact situation of the macular fibres in the chiasma should be mentioned such cases as those recorded by Nettleship ${ }^{2}$ of central amblyopia from tumour at the chiasma.

\section{Results obtained in the Optic Nerve.}

In all cases the degeneration is situated on the outer side of the nerve in front, while further back it reaches a central position which it maintains as far as the chiasma. The shape of the area in the anterior part of the nerve is triangular, the base being at the periphery, the apex at the central vessels.

\section{Results obtained in the Chiasma.}

The course of the macular fibres in the chiasma is as follows :-

Anteriorly in the chiasma they occupy the central area of both halves. Soon after entering the chiasma they pass towards the dorsum before any crossing bas occurred. The crossing takes place first at the dorsal periphery, at some distance behind the front of the chiasma. On proceeding backwards, though still partly remaining dorsal, it spreads from the dorsal surface in a ventral direction. The macular fibres never reach the ventral periphery.

As regards the question whether the macular fibres cross wholly or partially in the chiasma it is impossible from our experimeutal cases to make a definite statement, owing to the difficulty that exists in determining whether the wound was limited to the macular fibres or, on the other 
hand, whether it had damaged only a portion of these; while in Case 11 the decussation was complete, in Case 10 there were uncrossed fibres, even at the most posterior part of the chiasma. No help could be obtained in elucidating this point from Monkey 13, since in that case both optic nerves were affected.

With the view of studying by means of Marchi's method the degeneration resulting from tobacco poisoning we endeavoured to obtain this condition in two monkeys by feeding them with tobacco. The treatment lasted for six months in one case and for about one year in the other.

The optic nerves on examination showed in both cases a slight diffuse degeneration. This agrees with what was found by Barrett in his researches on the tobacco amblyopia of the horse in Australia. ${ }^{1}$

De Schweinitz ${ }^{2}$ failed to establish toxic (alcoholic) amaurosis in monkeys and dogs, probably, he adds, because the drug was not continued for a sufficiently long period.

Birch-Hirschfeld ${ }^{3}$ gave methyl-alcohol to seven rabbits and three fowls. In one of the rabbits a wedge-shaped temporal area was found immediately behind the globe, with partial destruction of the medullary sheaths. In the other rabbits and fowls there was no degeneration in the optic nerves. The same author ${ }^{4}$ found no changes in the optic nerves of four dogs to which methyl-alcohol had been given. Only one of three monkeys treated in the same way showed changes in the optic nerves, and the degeneration was limited to the anterior part of the nerves, it lay on the outer part and formed a wedge-shaped area. The rest of the nerves, the chiasma and tracts showed no sign of degeneration.

The pathological condition present in Monkey 13 corresponds more nearly to the ordinary type of lesion found

1 "System of Diseases of the Eye," Norris and Oliver, vol. iv., p. 817.

2 De Schweinitz, "System of Diseases of the Eye," Norris and Oliver, vol. iv., p. 809.

" Birch-Hirschfeld, “ Experimental Untersuchungen über die Pathogenese der Methyl-8lcoholamblyopie," Arch. für Ophthalmologie, 1901, zweites heft 358.

- Birch-Hirschfeld, "Weiterer Beitrag zur Pathogenese der Alcohol amblyopia," Arch. fur Ophthalmologie, 1902, Band liv., erstes Heft 68. 
in the optic nerves of man as a result of tobacco poisoning. ${ }^{1}$ We have endeavoured to get the previous history of this animal with reference to a tobacco habit, but have not succeeded in doing so.

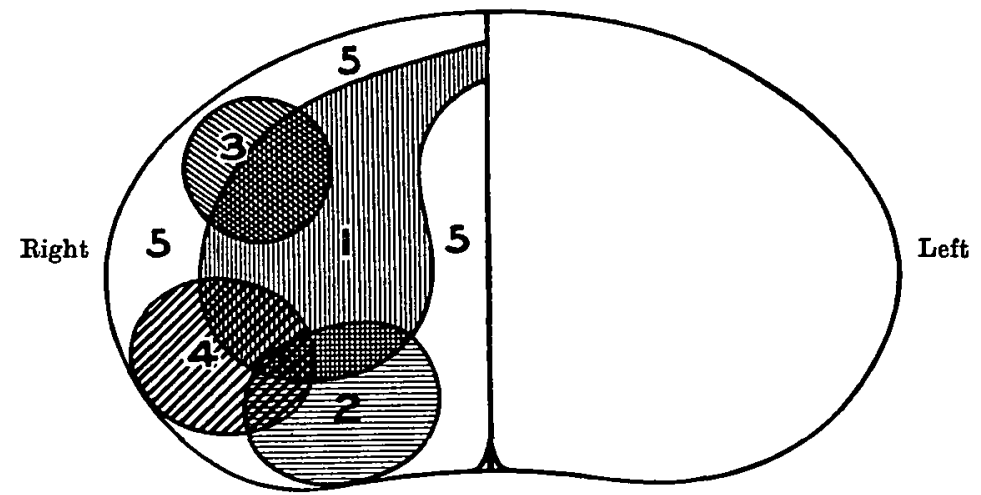

Front of chiasma. Seen from front.

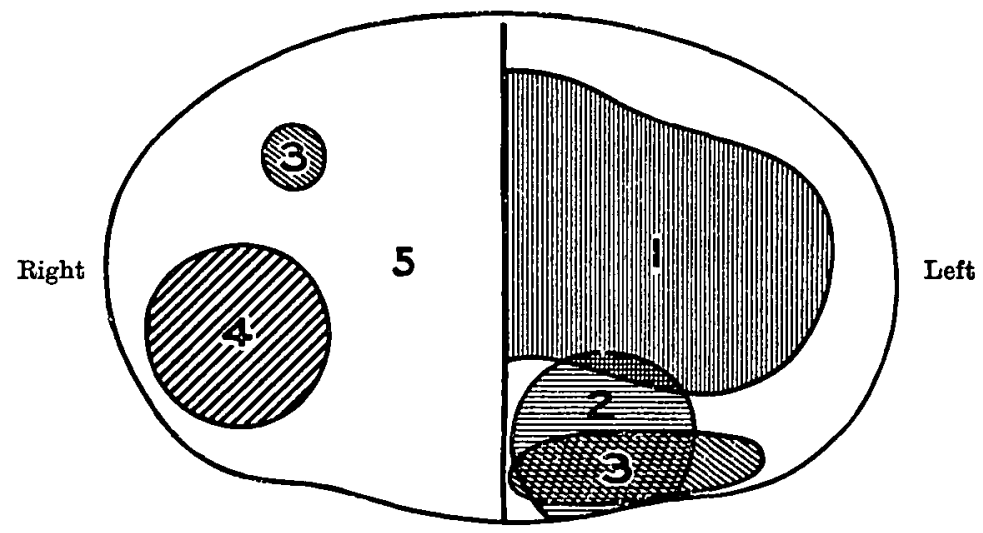

Back of chiasma. Seen from front.

Scheme of the situation of optic nerve-fibres in the chiasma in so far as they can be based on the results of the foregoing experiments.

(1) Macular fibres-Experiments 12, 10,5, and Case 13.

(2) Bundle of crossed fibres-Experiments 1, 2, 3, 4, 5, 8.

(3) Bundles of crossed and uncrossed fibres-Experiment 9.

(4) Bundle of uncrossed fibres-Experiments 6 and 7.

(5) A large number of uncrossed fibres occupying the clear area, as shown by Experiments 1, 2, 3, 4, 5 .

' Samelsohn, "Graefe-Saemisch Handbuch der gesammten Augenheilkunde," Auflage i., Bd. v., S. 834, 1877 ; Nettleship, Trans. Ophth. Soc., vol. i., 1882 ; Vossius, Archiv. für Ophthalmologie, Bd. xxviii., 3, 1883; Uhthoff, Graefe's Archiv., Bd. 


\section{Description of Plates.}

Fia. I illustrates the microscopical appearances of a coronal section through the most anterior part of a monkey's chiasma. The left optic nerve had been divided during life. The section was prepared by Marchi's method. The darkly-stained left half of the chiasma consists of degenerated fibres.

Fig. 2 illustrates the microscopical appearance of a coronal section from the anterior part of the same chiasma as is shown in figure 1, but further back in the chiasma. The section was prepared by Marchi's method. Degenerated fibres are seen crossing from the left half to the right half of the chiasma. Most of the crossing fibres are in the ventral part of the chissma and are arranged in transverse bands.

FIG. 3 illustrates the microscopical appearance of a portion of a coronal section from the anterior part of a monkey's chiasma. A lesion at the inner part of the right retina had been made during life. The section was prepared by Marchi's method. The whole of the darkly-stained degenerated area is shown in the plate, and is limited to the right half of the chiasma. A small septum is situated at the mid line ventrally.

Fig. 4 illustrates the microscopical appearances of a coronal section from further back in the same chiasma as is shown in figure 3 . Only a portion from the centre of the section is illustrated. This includes the whole of the darkly stained parts. The degenerated fibres are crossing in transverse bands from the right half to the left half of the chiasma.

FIG. 5 illustrates the microscopical appearances of a coronal section from a monkey's ohiasma. The papillo-macular fibres of the right eye had been injured during life. The section was prepared by Marchi's method. Degenerated fibres are passing at the dorsal periphery from the right half to the left half of the chissma. $D=$ dorsal, $V=$ ventral, $R=$ right, $L=$ left.

Figs. 6, 7 and 8 illustrate the microscopical appearances of coronal sections through the chiasma of a monkey. The sections were stained by Marchi's method. No. 6 is most anterior and No. 8 most posterior. The chiasma was from a monkey whose optic nerves showed degeneration corresponding exactly in situation to that found in tobacco amblyopia in man and in macular lesions in monkeys. The degeneration had not been produced by experiment. In figure 6 degeneration is seen at the centre of each half- on the right side the degeneration is seen to pass dorselly, and this is still more clearly shown on both sides in figure 7 . In figure 8 the degeneration, although still partly remaining dorsal, has spread in a ventral direction, but does not reach the ventral periphery. 